\title{
Philosophiques
}

\section{Olivier Reboul, Introduction à la rhétorique, Paris, P.U.F., coll. " Premier Cycle », 1991, VIII-238 pages.}

\section{Louis-André Dorion}

Volume 20, numéro 2, automne 1993

Perspectives sur la phénoménologie et l'intentionnalité

URI : https://id.erudit.org/iderudit/027240ar

DOI : https://doi.org/10.7202/027240ar

Aller au sommaire du numéro

Éditeur(s)

Société de philosophie du Québec

ISSN

0316-2923 (imprimé)

1492-1391 (numérique)

Découvrir la revue

Citer ce compte rendu

Dorion, L.-A. (1993). Compte rendu de [Olivier Reboul, Introduction à la rhétorique, Paris, P.U.F., coll. « Premier Cycle », 1991, VIII-238 pages.]

Philosophiques, 20(2), 508-511. https://doi.org/10.7202/027240ar d'utilisation que vous pouvez consulter en ligne.

https://apropos.erudit.org/fr/usagers/politique-dutilisation/ 
Olivier Reboul, Introduction à la rhétorique, Paris, P.U.F., coll. "Premier Cycle », 1991, VIII-238 pages.

par Louis-André Dorion

Cette Introduction à la rhétorique est l'un des tout premiers livres parus dans la nouvelle collection « Premier Cycle » qui propose, à l'intention des étudiants, des ouvrages d'initiation dans des domaines aussi variés que la philosophie, le droit, la littérature, l'économie, l'histoire, etc. Une fois complétée, cette collection comportera cent ouvrages. Pour ce qui est de la philosophie, on annonce la publication prochaine des Concepts fondamentaux de la philosophie, dont l'auteur est J.L. Marion, et d'une Histoire de la philosophie grecque, due à un groupe de spécialistes prestigieux (J. Barnes, L. Brisson, J. Brunschwig, M. 
Canto, G. Vlastos). Il ne fait aucun doute que ces ouvrages d'initiation, qui comblent l'une des lacunes les plus criantes de l'édition universitaire francophone, seront des plus profitables aux étudiants. O. Reboul (désormais R.) n'en est pas à son premier ouvrage d'introduction à la rhétorique, puisqu'on lui doit, sur le même sujet, un utile Que sais-je ? ( $\mathbf{n}^{\circ}$ 2133) dont la première édition remonte à ig84. Même s'il « s'inspire » parfois fortement de certains exposés du Que sais-je? - ce qui est somme toute inévitable -, le présent ouvrage est loin de faire double emploi, puisque non seulement il contient de nombreux développements inédits, mais il traite aussi plus en profondeur des sujets abordés peut-être un peu rapidement - mais c'est la loi du genre ! - dans le Que sais-je?

Dans l'introduction, R. rappelle à juste titre que la rhétorique, après avoir été longtemps décriée et même supprimée de l'enseignement, a finalement été réhabilitée dans les années soixante par de nombreux et influents travaux, dont ceux de C. Perelman et $\mathrm{L}$. Olbrechts-Tyteca. Ces derniers renouent avec la tradition qui voit dans la rhétorique l'art d'argumenter, alors que les études de G. Genette et du «Groupe Mu » font plutôt de la rhétorique l'étude du style, et notamment des figures. Quoiqu'il cherche à dégager l'élément commun à ces deux approches en apparence complètement étrangères l'une à l'autre, élément commun qui serait « l'articulation des arguments et du style dans une même fonction » (p. 4), R. se montre résolument classique, et partant plus proche de Perelman, en définissant la thétorique comme « l'art de persuader par le discours » (p. 4). On se rappelle que Gorgias disait déjà de la rhétorique qu'elle était « ouvrière de persuasion » (cf. Gorgias 453a, 455a). Avant d'aborder l'étude de la rhétorique par le biais de son histoire, R. en identifie les quatre principales fonctions. En vertu de sa définition, la rhètorique a premièrement une fonction persuasive. Soit ; mais par quels moyens le discours est-il persuasif ? R. montre clairement que cette force persuasive comporte deux aspects, l'un argumentatif, l'autre oratoire, qu'il n'est pas toujours aisé de distinguer. La deuxième fonction de la rhétorique est de nature herméneutique, c'est-à-dire que la rhétorique a pour tâche, non seulement de produire des discours, mais aussi de les interpréter. Troisièmement, la rhétorique a une importante fonction heuristique, c'est-à-dire de découverte. Cela peut surprendre, tant nous, modernes, avons naturellement tendance à faire de la découverte le privilège exclusif de la science. R. rappelle toutefois à juste titre que « nous vivons dans un monde qui n'est pas entièrement justiciable de la connaissance scientifique, un monde où la vérité est rarement évidente et la prévision certaine rarement possible » (p. 1o). C'est précisément dans les nombreux domaines rebelles à la démonstration scientifique que la rhétorique est appelée à jouer son irremplaçable fonction heuristique : « Dans un monde sans évidence, sans démonstration, sans prévision certaine, dans notre monde humain, la rhétorique a pour rôle, en défendant telle ou telle cause, d'éclairer celui qui doit trancher. Elle contribue, là où nulle solution n'est écrite d'avance, à inventer une solution. Et elle le fait en instaurant un débat contradictoire, que seuls ses "procédés" rendent possible, qui sans eux tomberait vite dans le tumulte et la violence » (p. II).

Le corps de l'ouvrage se divise en neuf chapitres, dont nous donnerons ici un bref aperçu. Les deux premiers chapitres se penchent sur les origines grecques de la rhétorique. Le premier chapitre (p. 13-22) retrace les débuts 
siciliens de la rhétorique et expose bien les raisons pour lesquelles celle-ci suscita très vite un débat qui opposa Platon aux sophistes et à Isocrate. Le deuxième chapitre (p. 33-54), qui est un peu plus technique, mais qui n'en demeure pas moins parfaitement clair et accessible, traite de la difficile question des rapports entre la dialectique et la rhétorique dans la philosophie d'Aristote. Après cet aperçu des origines de la rhétorique, et des premières réflexions sur sa nature, ses fonctions et ses limites, $\mathrm{R}$. consacre le troisième chapitre au système rhétorique (p. 55-80), tel qu'il a été codifié par les rhéteurs latins, notamment Quintilien ( $\mathrm{I}^{\mathrm{er}}$ siècle ap. J.C.). Dans sa forme canonique, ce système comporte quatre parties : l'invention [heuresis], la disposition [taxis|, l'élocution llexisl et l'action Ihypocrisis], que R. présente de façon détaillée. La partie historique de cet ouvrage se termine avec le chapitre IV (p. 81-98), qui présente une histoire, nécessairement rapide, de la rhétorique depuis le Ir siècle ap. J.C. jusqu'à aujourd'hui. R. consacre de belles pages à l'analyse des similitudes et des différences entre la rhétorique et la publicité et il revient plus en profondeur sur les raisons multiples, évoquées au début de l'ouvrage, qui ont présidé à la réhabilitation de la rhétorique au début des années soixante. Les chapitres V à IX concernent plus directement le discours rhétorique lui-même. Ainsi le chapitre V (p. 99-ı2o) s'efforce-t-il de dégager les principales caractéristiques de l'argumentation rhétorique. Selon R., le fait que le discours rhétorique allie, pour persuader, une composante argumentative à une composante oratoire, donc un contenu et une forme, entraîne deux principales conséquences : a) le discours rhétorique est « non paraphrasable », c'est-à-dire qu'on « ne peut le traduire, même dans sa propre langue, par un discours ayant tout à fait le même sens » (p. IIo) ; b) le propre du discours rhètorique est également d'être fermé, de ne pas admettre de réplique : « on ne peut réfuter une rhétorique que sur son propre plan, que par une autre rhétorique » (p. IIo). Le chapitre VI (p. I2I-I42) présente les principales figures de style qui composent l'arsenal rhétorique. R. les classe en figures de mots (allitération, antanaclase, etc.), figures de sens (métonymie, métaphore, synecdoque, etc.), figures de construction (ellipse, asyndète, anacoluthe, etc.) et, enfin, figures de pensée (ironie, prosopopée, etc.). Etant donné que le lecteur non helléniste risque d'être quelque peu dérouté par l'« exotisme » des noms qui désignent ces figures (comme « hyperbate », « apodioxie », « chleuasme », « épanorthose » et autres « aposiopèses »), R. a eu la délicatesse d'ajouter un précieux index et glossaire des termes techniques (p. 230-238) employés dans l'ouvrage. Le chapitre VII (p. 143-ı64) précise la fonction herméneutique de la rhétorique en exposant les principales questions que le lecteur doit adresser au texte. Cette lecture rhétorique des œuvres est enrichie par les indications fournies au chapitre suivant (p. I65-194) sur la façon de repérer et d'identifier les différents arguments employés et déployés dans un texte. Enfin, ces éléments d'une herméneutique rhétorique sont en quelque sorte mis en pratique dans le dernier chapitre (p. 195-222), où l'on propose des exemples de lecture rhétorique. R. analyse les procédés rhétoriques mis en œuvre dans sept courts textes, empruntés pour les uns à des auteurs classiques (Corneille, Rousseau), pour d'autres à une forme beaucoup moins relevee de prose, comme des histoires yiddish ou encore une interview accordée par F. Dolto au journal Libération. Particulièrement réussi est l'exercice de lecture qui porte sur un passage du Discours de la 
méthode de Descartes. R. n'a aucune peine à dévoiler les différents procédés rhétoriques auxquels fait appel un auteur qui, à l'en croire, était un ennemi déclaré de la rhétorique.

Ce livre n'est évidemment pas sans défaut et l'on pourrait toujours contester, ici ou là, certains points de l'exposé. Par exemple, l'interprétation que donne R. (p. 20) de la célèbre formule « l'homme est la mesure de toutes choses » fait de Protagoras un relativiste radical, ce qui revient à jeter injustement le discrédit sur une pensée qui ètait bien au fait des écueils insurmontables liés au relativisme absolu. Ou encore, il est inexact d'affirmer (p. 43) que l'une des règles de la dialectique aristotélicienne est de ne poser que des questions qui admettent une réponse par « oui » ou « non ». Cette règle était plutôt le fait des éristiques, et Aristote invite même le répondant à l'enfreindre, pour éviter qu'il ne tombe dans les pièges qu'une application stricte de cette règle peut entraîner (cf. Réfutations sophistiques 17, I75b7-I4). Le live renferme bien quelques autres inexactitudes de ce genre, mais il s'agit, comme on aura pu le constater, de détails qui n'entament en rien la qualité, la clarté et la rigueur d'un exposé qui, rappelons-le, est avant tout conçu comme un ouvrage d'introduction destiné aux étudiants de premier cycle. Ne boudons donc pas notre plaisir et souhaitons que tous les volumes à paraître dans cette nouvelle collection soient aussi stimulants.

Département de philosophie

Université de Montréal 